\title{
2MASS J15491331-3539118: a new low-mass wide companion of the GQ Lup system ${ }^{\star}$
}

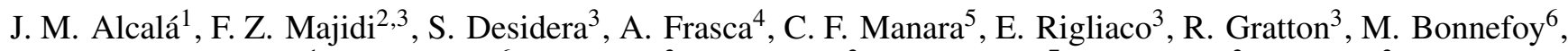 \\ E. Covino ${ }^{1}$, G. Chauvin ${ }^{6}$, R. Claudi ${ }^{3}$, V. D'Orazi ${ }^{3}$, M. Langlois ${ }^{7}$, C. Lazzoni ${ }^{3}$, D. Mesa ${ }^{3}$, \\ J. E. Schlieder ${ }^{8}$, and A. Vigan ${ }^{9}$
}

1 INAF-Osservatorio Astronomico di Capodimonte, via Moiariello 16, 80131 Napoli, Italy e-mail: juan.alcala@inaf.it

2 Dipartimento di Fisica, dell'Universitá di Roma Sapienza, P.le A. Moro 5, Roma 00185, Italy

3 INAF-Osservatorio Astronomico di Padova, vicolo dell'Osservatorio 5, 35122 Padova, Italy

4 INAF-Osservatorio Astrofisico di Catania, via S. Sofia 78, 95123 Catania, Italy

5 European Southern Observatory, Karl-Schwarzschild-Str. 2, 85748 Garching bei Munchen, Germany

${ }^{6}$ Université Grenoble Alpes, CNRS, IPAG, 38000 Grenoble, France

7 CRAL, UMR 5574, CNRS, Université de Lyon, École Normale Supérieure de Lyon, 46 Allée d'Italie, 69364 Lyon Cedex 07, France

8 Exoplanets and Stellar Astrophysics Laboratory, Code 667, NASA Goddard Space Flight Center, Greenbelt, MD 20771, USA

9 Aix Marseille Université, CNRS, CNES, LAM, Marseille, France

Received 12 December 2019 / Accepted 30 January 2020

\begin{abstract}
Substellar companions at wide separation around stars hosting planets or brown dwarfs (BDs) yet close enough for their formation in the circumstellar disc are of special interest. In this Letter we report the discovery of a wide (projected separation $\sim 16^{\prime \prime} .0$, or $2400 \mathrm{AU}$, and position angle $114.61^{\circ}$ ) companion of the GQ Lup A-B system, most likely gravitationally bound to it. A VLT/Xshooter spectrum shows that this star, 2MASS J15491331-3539118, is a bonafide low-mass $\left(\sim 0.15 M_{\odot}\right)$ young stellar object (YSO) with stellar and accretion/ejection properties typical of Lupus YSOs of similar mass, and with kinematics consistent with that of the GQ Lup A-B system. A possible scenario for the formation of the triple system is that GQ Lup A and 2MASS J15491331-3539118 formed by fragmentation of a turbulent core in the Lup I filament, while GQ Lup B, the BD companion of GQ Lup A at $0^{\prime \prime}$.7, formed in situ by the fragmentation of the circumprimary disc. The recent discoveries that stars form along cloud filaments would favour the scenario of turbulent fragmentation for the formation of GQ Lup A and 2MASS J15491331-3539118.
\end{abstract}

Key words. stars: pre-main sequence - stars: low-mass - accretion, accretion disks - protoplanetary disks

\section{Introduction}

It is now well established that most stars form in binaries or higher-order multiple systems (Monin et al. 2007, and references therein). Substellar wide companions around stars that host planets or brown dwarfs (BDs) at separation close enough for formation in the circumstellar disc are of special interest. The dynamical environment of young binaries and multiple systems may have an important impact on disc evolution (Kurtovic et al. 2018; Manara et al. 2019) and therefore on planet formation. During the early evolution phase, the individual discs of substellar companions, including those in the planetary-mass regime, accrete additional material from the gas-rich parent disc, and therefore their discs are more massive and their accretion rates higher than objects of similar mass formed in isolation. This means that disc masses and accretion rates of these very lowmass companions are expected to be independent of the mass of the central substellar object, and higher than predicted from the $\dot{M}_{\text {acc }} \propto M_{\star}{ }^{2}$ scaling relation for more massive young stellar

\footnotetext{
* Based on observations collected at the European Southern Observatory at Paranal, under program 103.C-0200(A), and archive data from 074.C-0037(A) and 082.C-0390(A).
}

objects (YSOs). These arguments were used by Stamatellos \& Herczeg (2015) to explain the very high levels of accretion observed in substellar and planetary-mass companions at wide orbits in some T Tauri stars (Zhou et al. 2014).

The results of ALMA observations of wide multiple systems (Williams et al. 2014; Kurtovic et al. 2018; Manara et al. 2019) have provided important information on the disc morphologies and geometry of these systems, revealing misalignments of the disc and orbital angular momentum vectors, which provide important clues about the formation mechanisms of these systems. Likewise, the discovery of ultra-wide pairs (UWPs; 1000-6000 AU; Joncour et al. 2017) is shedding light on the formation mechanisms at separation scales of the pristine star forming cores.

The Gaia DR2 catalogue (Gaia Collaboration 2018) offers the opportunity to study the kinematic and astrometric properties of large samples of stars on large sky areas, allowing the selection of comoving YSO candidates in star forming regions with similar parallaxes and kinematics to those of bonafide YSOs. As part of an investigation (Majidi et al., in prep.) aimed at characterising companions to SPHERE targets hosting giant planets and discs, we searched for wide companions associated to 
SHINE SPHERE-GTO targets (Chauvin et al. 2017) in the Gaia DR2, finding several tens of candidates. We focused on a few objects around stars of high specific interest due to the presence of planetary or BD companions and spatially resolved circumstellar discs. GQ Lup, a $\sim 1 M_{\odot}$ (Alcalá et al. 2017; MacGregor et al. 2017) classical T Tauri star, has both a spatially resolved circumstellar disc (MacGregor et al. 2017) and a BD companion (GQ Lup B; Neuhäuser et al. 2005) with a mass about 20-40 $M_{\text {jup }}$ at a separation of 0 !'70. This BD companion was found to be actively accreting (Zhou et al. 2014), suggesting the presence of a disc around it, although more recent observations show that the accretion is non-steady (Wu et al. 2017).

In this Letter we report the discovery of a wide companion of the GQLup system that is most likely gravitationally bound, making it a very interesting triple system. In Sect. 2, the target selection and observations with the VLT/X-shooter spectrograph are described. In Sect. 3, we characterise the new companion in terms of its stellar physical and accretion/ejection parameters. In Sect. 4, we discuss the nature of GQLup as a triple system and the implications on its possible formation mechanism.

\section{Target selection and observations}

We searched the Gaia DR2 catalogue for candidates within 5 arcmin of the selected targets with similar parallaxes and proper motions. We identified a companion candidate ${ }^{1}$ at $16^{\prime \prime} 0$ of GQLup and position angle $114.61^{\circ}$. This candidate is identified with 2MASS J15491331-3539118 and is slightly fainter (by $0.2-0.5 \mathrm{mag}$ ) in the $J, H$, and $K$ bands $(14.85,14.08$ and 13.82 , respectively) than GQLup B. Its mass was then expected to be slightly lower ( $\sim 20 M_{\text {jup }}$ for $2 \mathrm{Myr}$ age) than GQLup B. From its near-infrared (NIR) colours and position on a $J-H$ versus $H-K$ diagram, the object appears moderately reddened ( $\left.A_{V} \sim 0.5-1 \mathrm{mag}\right)$ and does not show strong NIR excess. However, whether the discovered source is gravitationally bound to the GQLup system remains inconclusive. Therefore, although it could be referred to as GQLupC, for caution we refer to it as 2MASS J15491331, and for the reasons discussed in Appendix B, we adopt the distance of $152( \pm 1)$ pc from the Gaia DR2 as for GQLup A (Gaia Collaboration 2018).

We investigated 2MASS J15491331 using the VLT/Xshooter spectrograph (Vernet et al. 2011). Service mode observations were conducted during the night of 28 May 2019. We adopted the same observational and data processing strategies as in previous investigations of YSOs in Lupus (see Alcalá et al. 2014, 2017, and Appendix A for the details). Figure 1 shows the flux distribution of the reduced X-shooter spectrum of 2MASS J15491331 compared with the photometric fluxes available in the Gaia DR2 and 2MASS. The flux calibration of the spectrum yields results consistent with the photometric fluxes within the flux calibration uncertainties of $15-20 \%$.

\section{Results}

\subsection{The spectrum of 2MASS J15491331}

The spectrum of 2MASS J15491331 is typical of a late-type YSO showing a wide (WH $\alpha(10 \%) \sim 510 \mathrm{~km} \mathrm{~s}^{-1}$ ) and strong $(E W \sim 100 \AA) \mathrm{H} \alpha$ emission, and the Li I (6708 $\AA$ ) absorption line (See Fig. C.1), as well as numerous permitted and forbidden emission lines, the latter being of comparable intensity to

\footnotetext{
1 By wide companion candidate we refer to the object spatially proximate to the GQ Lup system, with a projected separation of a few tens of arcsec, but not necessarily gravitationally bound to the system.
}

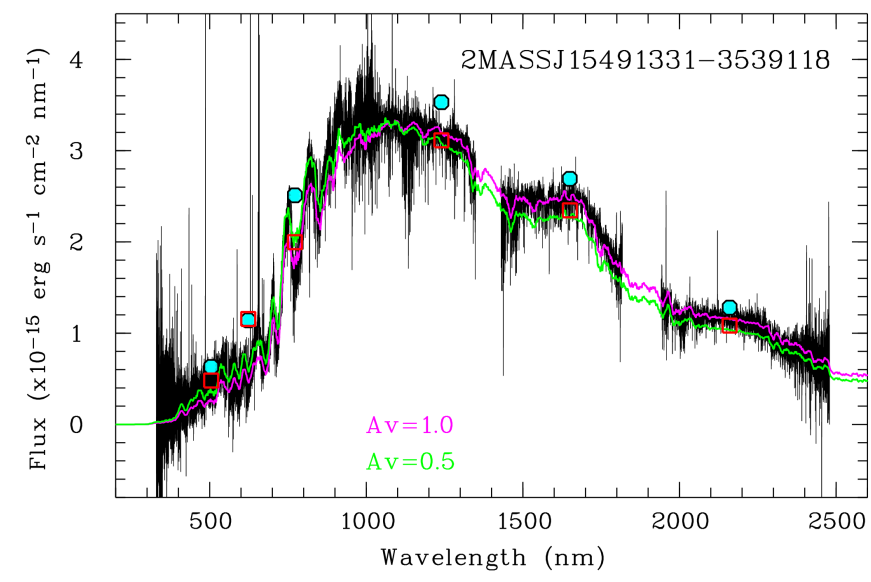

Fig. 1. X-shooter spectrum of 2MASS J15491331 (in black) compared with photometric fluxes (light blue dots) from the Gaia DR2 and 2MASS catalogues. Synthetic fluxes derived from the observed spectrum are shown as red squares. The lines show a BT-Settl model of the same effective temperature as the target, for the two reddening values indicated in the labels. The model has been normalised to the flux of 2MASS J15491331 at $830 \mathrm{~nm}$.

the former. This behaviour may be explained by a contrast effect possibly due to obscuration of the star and of the accretion flows by the inner disc regions; it is similar to the one observed in the spectrum of Par-Lup 3-4, a subluminous, edge-on YSO in the Lupus III cloud (Alcalá et al. 2014, 2017), and suggests that we are viewing 2MASS J15491331 at a high inclination angle close to edge-on. The Balmer decrement is also consistent with such a geometry (See Appendix D). Many Balmer lines and several $\mathrm{He} \mathrm{I}$ and $\mathrm{Ca}$ II lines and the $\mathrm{Pa} \beta$ and $\mathrm{Br} \gamma$ lines are observed in emission. We were able to measure the flux of the Balmer lines up to $\mathrm{H} 15$, as well as of several other emission lines (see Appendix C). Despite a low $\mathrm{S} / \mathrm{N}$ in the $\mathrm{UV}$, U-excess continuum emission in 2MASS J15491331 is also evident (see Fig. C.2). All these results confirm that the object is a bonafide YSO with high mass accretion and ejection rates. In the following we use the X-shooter spectrum combined with the Gaia DR2 data to characterise the object.

\subsection{Stellar parameters}

The physical stellar parameters given in Table 1 were derived using the same methods applied for our previous X-shooter surveys in star forming regions (Alcalá et al. 2014, 2017; Manara et al. 2017). The details are given in Appendix B. The same methods yield a very similar extinction value $\left(A_{V}=0.70 \mathrm{mag}\right.$, Alcalá et al. 2017) for GQ Lup A. A fit of a BT-Settl model spectrum (Allard et al. 2012) of the same $T_{\text {eff }}$ as the object suggests a moderate extinction of $A_{V} \sim 1.0 \mathrm{mag}$ for 2MASS J15491331 (see Fig. 1), which is consistent with the value derived using our methods.

Unexpectedly, the resulting spectral type M4 indicates that 2MASS J15491331 is a star, and not a BD. We note that the luminosity derived from the bolometric flux of the star (see Appendix B) is more than an order of magnitude lower than that of Lupus YSOs of similar $T_{\text {eff }}$, again suggesting subluminosity due to obscuration of the inner disc regions. This may also explain the faint NIR magnitudes in comparison with Lupus

\footnotetext{
2 We note that Petrus et al. (2020) show that extinction may be overestimated by the BT-Settl models.
} 
Table 1. Physical stellar parameters of 2MASS J15491331.

\begin{tabular}{lccccc}
\hline \hline SpT & $\begin{array}{c}A_{V} \\
(\mathrm{mag})\end{array}$ & $\begin{array}{c}T_{\text {eff }} \\
(\mathrm{K})\end{array}$ & $\begin{array}{c}L_{\star} \\
\left(L_{\odot}\right)\end{array}$ & $\begin{array}{c}R_{\star} \\
\left(R_{\odot}\right)\end{array}$ & $\begin{array}{c}M_{\star} \\
\left(M_{\odot}\right)\end{array}$ \\
\hline
\end{tabular}

\begin{tabular}{llllll}
$\mathrm{M} 4 \pm 0.5$ & $1.0 \pm 0.5$ & $3190 \pm 100$ & $0.004 \pm 0.001$ & $0.21 \pm 0.06$ & $0.15 \pm 0.05$ \\
\hline
\end{tabular}

Table 2. Parameters derived from ROTFIT.

\begin{tabular}{lccccc}
\hline \hline $\begin{array}{l}T_{\text {eff }} \\
(\mathrm{K})\end{array}$ & $\log g$ & $\begin{array}{c}R_{\star}(\dagger) \\
\left(R_{\odot}\right)\end{array}$ & $\begin{array}{c}L_{\star}(\dagger) \\
\left(L_{\odot}\right)\end{array}$ & $\begin{array}{c}R V \\
\left(\mathrm{~km} \mathrm{~s}^{-1}\right)\end{array}$ & $\begin{array}{c}v \sin i \\
\left(\mathrm{~km} \mathrm{~s}^{-1}\right)\end{array}$ \\
\hline $3230 \pm 101$ & $3.74 \pm 0.23$ & $0.87 \pm 0.20$ & $0.07 \pm 0.02$ & $-2.0 \pm 2.8$ & $13.0 \pm 6.0$ \\
\hline
\end{tabular}

Notes. ${ }^{(\dagger)}$ Values derived from $\log g$ and $M_{\star}$.

objects of similar spectral type. The stellar radius, $R_{\star}$, derived from $L_{\star}$ and $T_{\text {eff }}$ is also underestimated.

The ROTFIT code (Frasca et al. 2015) applied to the $\mathrm{X}$-shooter spectrum yields a $T_{\text {eff }}$ which is in excellent agreement with the value derived from the spectral type and surface gravity typical of low-mass PMS stars. The results are provided in Table 2 . The radial velocity $(R V)$ is consistent, within errors, with the values in the literature for the GQ Lup system. ROTFIT operates on spectral segments normalised to unity, and therefore a measure of $A_{V}$ is not provided by the code.

An estimate of $R_{\star}$ that is independent of $L_{\star}$ can be obtained using the ROTFIT $\log g$ value and assuming the mass of $0.15 M_{\odot}$, which is not affected by subluminosity because the PMS tracks run almost vertically at the $T_{\text {eff }}$ values close to that of 2MASS J15491331. When doing so, we obtain a radius of $0.87 R_{\odot}$ for the star and hence a luminosity that is higher by a factor of $\sim 17$ than the estimated $L_{\star}$ based on the bolometric flux. This places 2MASS J15491331 on the Hertzsprung-Russell (HR) diagram in good agreement with other Lupus members, with an estimated age of 2 Myr based on the Baraffe et al. (2015) theoretical isochrones (See Fig. B.1), and consistent within errors with the age of the GQLup system of $\sim 1 \mathrm{Myr}$ (Frasca et al. 2017).

\subsection{Mass accretion and ejection}

The analysis to derive the accretion luminosity, $L_{\text {acc }}$, which measures the Balmer continuum excess emission with respect to the photospheric one, is described in Appendix C. We estimated $\log \left(L_{\text {acc }} / L_{\odot}\right)=-3.54$. We also derived $L_{\text {acc }}$ from the luminosity, $L_{\text {line }}$, of a number of Balmer and other emission lines using the $L_{\text {line }}-L_{\text {acc }}$ relationships (Alcalá et al. 2017). From this analysis we calculated $\log L_{\text {acc }}=-3.74 \pm 0.20$, which is in good agreement with the value derived from the Balmer continuum fit (see Appendix C for details). However, we note that this is about one order of magnitude lower than the typical $L_{\text {acc }}$ of Lupus YSOs of similar mass; the reason is again that the emission from the accretion funnels is obscured by the edge-on inner-disc regions. Therefore, as in other cases of subluminous YSOs (Alcalá et al. 2014, 2017), we use the luminosity of the [O I] $\lambda 6300 \AA$ forbidden line to correct for these effects, deriving $\log L_{\text {acc }}=-2.85 \pm 0.20$ (see Appendix $\mathrm{C}$ for details). The correction factor is very similar to the one on $L_{\star}$, which is reasonable as the accretion funnels should be obscured by the inner disc to a similar extent to the stellar photosphere. The corrected accretion luminosity can be converted into a mass accretion rate using Eq. (C.1) and the ROTFIT corrected $R_{\star}$ value, yielding $\log \dot{M}_{\text {acc }}=-9.50$ or $\dot{M}_{\text {acc }}=3.3 \times 10^{-10} M_{\odot} \mathrm{yr}^{-1}$, which is within the typical range of values for Lupus YSOs of comparable mass.

The mass loss rate was estimated following Natta et al. (2014, see Appendix C for details). The value we derive is $M_{\text {loss }} \sim 7.0 \times 10^{-12} M_{\odot} \mathrm{yr}^{-1}$, similar to the values for very lowmass YSOs, and implying a $M_{\text {loss }} / \dot{M}_{\text {acc }}$ ratio of $\sim 0.02$ in the expected range for magneto-centrifugal jet launch models $(0.01$ $<M_{\text {loss }} / \dot{M}_{\text {acc }}<0.5$ ) and studies of accreting YSOs (Cabrit et al. 2009; Whelan et al. 2014; Nisini et al. 2018, and references therein).

\subsection{Kinematics}

The radial velocity estimates in the literature for GQLup A are $-3.6 \pm 1.3 \mathrm{~km} \mathrm{~s}^{-1}$ (Frasca et al. 2017) and $-2.8 \pm 0.2 \mathrm{~km} \mathrm{~s}^{-1}$ (Schwarz et al. 2016). We also retrieved ten spectra taken with HARPS (Program ID 074.C-0037(A) and 082.C-0390(A)) from the ESO archive. The RVs drawn from the instrument pipeline yield an average of $-3.3 \mathrm{~km} \mathrm{~s}^{-1}$ with rms scatter of $0.8 \mathrm{~km} \mathrm{~s}^{-1}$ (dominated by activity-induced RV jitter) over a baseline of 1470 days. The ROTFIT RV measurement of $-2.0 \pm 2.8 \mathrm{~km} \mathrm{~s}^{-1}$ for 2MASS J15491331 is consistent, within errors, with these values. Likewise, the Gaia DR2 proper motion for 2MASS J15491331 $\left(\mu_{\alpha} \cos \delta=-14.81 \pm 0.97\right.$ mas and $\mu_{\delta}=-21.95 \pm 0.65$ mas) is consistent within $\sim 1 \sigma$ with that of GQLup A $\left(\mu_{\alpha} \cos \delta=-14.26 \pm 0.10\right.$ mas and $\mu_{\delta}=-23.60 \pm$ 0.07 mas). Therefore, the kinematics of 2MASS J15491331 is highly consistent with that of the GQLup system.

\section{Discussion and conclusions}

Above, we demonstrate that 2MASS J15491331 is a bonafide low-mass YSO in the Lupus I cloud, with stellar, accretion, and ejection properties typical of Lupus YSOs of similar mass, and kinematics consistent with that of the GQLup A-B system. At the distance of the GQ Lup system, 16".0 corresponds to a separation of about $2430 \mathrm{AU}$. Multiples with such separations are not expected to be very common (Baron et al. 2018), but if 2MASS J15491331 is physically bound to GQ Lup A it would be another example of a young low-mass wide-bound system (see Petrus et al. 2020, for other cases), and also an interesting case for further studies with high-resolution millimetre observations with ALMA (see Williams et al. 2014; Kurtovic et al. 2018; Manara et al. 2019, for ALMA observations in similar systems).

The separation of $2430 \mathrm{AU}$ of 2MASS J15491331 is larger than that of the wide pairs so far studied with ALMA, but it is in the low tail of the separation distribution (1000-60000 AU) for UWPs recently detected by Joncour et al. (2017) in the Taurus star forming region. As noted in that work, the UWPs with separations of $<5000 \mathrm{AU}$ have been found to be physical wide binaries (Kraus \& Hillenbrand 2009). This would favour the conclusion that GQLup A-B-2MASS J15491331 is likely a gravitationally bound system. It is difficult to firmly establish whether 2MASS J15491331 is gravitationally bound to GQ Lup with our data alone. The escape velocity at such a large separation is less than $1 \mathrm{~km} \mathrm{~s}^{-1}$, which is comparable with the expected rms value due to turbulence and well below the accuracy of the current estimates of the relative motions. However, GQLup A and 2MASS J15491331 are likely still gravitationally bound. In fact, at a velocity of $1 \mathrm{~km} \mathrm{~s}^{-1}$, a star travels $1 \mathrm{pc}$ in $1 \mathrm{Myr}$, roughly the estimated age of the GQLup system (Frasca et al. 2017); 1 pc is $\sim 22$ arcmin at the distance of GQLup, which is two orders of magnitude more than the separation of the GQLup system components. Moreover, the probability of a chance projection is 
very small, and can be ruled out based on the statistical grounds presented in Appendix E.

The inclination $i=60.2^{\circ}$ of the circumprimary disc (MacGregor et al. 2017; Ansdell et al. 2018) and the edge-on geometry of the 2MASS J15491331 disc might in principle be an indication that the angular momentum vectors are not aligned. Misalignments have been observed in other multiple systems with components at closer separations than 2MASS J15491331 (Brinch et al. 2016; Kurtovic et al. 2018; Williams et al. 2014; Jensen \& Akenson 2014; Manara et al. 2019). These studies provide important clues for the mechanisms leading to misalignments during the early evolutionary phase, and discuss some possible scenarios, such as for example fragmentation of a turbulent core where the directions of the angular momentum vectors change. This is supported by hydrodynamical simulations (Bate 2018) that indicate misaligned discs are mainly due to fragmentation in turbulent environments and stellar capture; alternatively, the dynamical interactions of three or more protostars may lead to random changes of the orbital axes during the early Class 0 -I phases. Also, the environmental conditions in which the discs are formed may affect the alignments with a variety of disc orientations being the result of gas accreted from the parent cloud with a different angular momentum. With our data alone it is not possible to establish the relative geometries of the GQ Lup A-B -2MASS J15491331 discs. However, evidence for the alignment of the system will be presented in a follow-up paper (Lazzoni et al., in prep.). We also note that the orbital analysis by Schwarz et al. (2016) suggests a $\sim 60^{\circ}$ inclined orbit for GQ Lup B, that is, very similar to that of the circumprimary disc.

A firm conclusion on the formation mechanism of the GQ Lup A-B-2MASS J15491331 system would be premature. Given its high mass-accretion rate, the in situ formation of GQ Lup B by circumprimary disc fragmentation (Stamatellos \& Herczeg 2015) cannot be ruled out (Wu et al. 2017; MacGregor et al. 2017), although the presence of 2MASS J15491331 might have had an impact on its orbit due to dynamical interaction. Joncour et al. (2017) concluded that the young UWPs may be pristine imprints of their spatial configuration at birth resulting from sequential fragmentation of the natal molecular core. Therefore, a likely scenario for the formation of the GQ Lup A-B-2MASS J15491331 system may be that GQ Lup A and 2MASS J15491331 formed by fragmentation of a turbulent core, with GQ Lup B formed in situ by the fragmentation of the circumprimary disc. The recent result that stars form along filaments (e.g. Benedettini et al. 2018, and references therein; see also Appendix E) would favour the scenario of turbulent fragmentation for the formation of GQ Lup A and 2MASS J15491331. Further high-resolution interferometric observations of wide multiple systems with discs in millimetre bands will shed light on their formation mechanisms.

Acknowledgements. We thank the anonymous referee for his/her comments and suggestions. This work has been supported by the project PRIN-INAF 2016 The Cradle of Life - GENESIS-SKA "General Conditions in Early Planetary Systems for the rise of life with SKA", and by the project PRIN-INAF-MAINSTREAM 2017 "Protoplanetary disks seen through the eyes of new-generation instruments". This project has received funding from the European Union's Horizon 2020 research and innovation programme under the Marie SklodowskaCurie grant agreement No 823823 (DUSTBUSTERS). This work was partly supported by the Deutsche Forschungs-Gemeinschaft (DFG, German Research Foundation) - Ref no. FOR 2634/1 TE 1024/1-1. This research made use of the SIMBAD database, operated at the CDS (Strasbourg, France). This work has made use of data from the European Space Agency (ESA) mission Gaia (https://www.cosmos.esa.int/gaia), processed by the Gaia Data Processing and Analysis Consortium (DPAC, https://www.cosmos.esa.int/ web/gaia/dpac/consortium). Funding for the DPAC has been provided by national institutions, in particular the institutions participating in the Gaia Multilateral Agreement.

\section{References}

Alcalá, J. M., Natta, A., Manara, C., et al. 2014, A\&A, 561, A2 Alcalá, J. M., Manara, C., Natta, A., et al. 2017, A\&A, 600, A20 Alcalá, J. M., Manara, C., France, K., et al. 2019, A\&A, 629, A108 Allard, F., Homeier, D., \& Freytag, B. 2012, Trans. R. Soc. London Ser. A, 370, 2765

Ansdell, M., Williams, J. P., Trapman, L., et al. 2018, ApJ, 859, 21 Antoniucci, S., Nisini, B., Giannini, T., et al. 2017, A\&A, 599, A105 Baraffe, I., Homeier, D., Allard, F., \& Chabrier, G. 2015, A\&A, 577, A42 Baron, F., Artigau, É., Rameau, J., et al. 2018, AJ, 156, 137

Bate, M. R. 2018, MNRAS, 475, 5618

Benedettini, M., Pezzuto, S., Schisano, E., et al. 2018, A\&A, 619, 52 Brinch, C. H., Jorgensen, J. K., Hogerheijde, M. R., et al. 2016, ApJ, 830, L16 Cabrit, S. 2009, in Protostellar Jets in Context, Astrophysics and Space Science Proceedings Series, eds. K. Tsinganos, T. Ray, \& M. Stute (Berlin: Springer), 247

Cardelli, J. A., Clayton, G. C., \& Mathis, J. S. 1989, ApJ, 345, 245

Chauvin, G., Desidera, S., Lagrange, A., et al. 2017, in SF2A-2017: Proc. of the Ann. Meet. of the French Society of Astronomy and Astrophysics, held 4-7 July, 2017 in Paris, eds. C. Reylé, P. Di Matteo, F. Herpin, et al., 331 Frasca, A., Biazzo, K., Lanzafame, A. C., et al. 2015, A\&A, 575, A4 Frasca, A., Biazzo, K., Alcalá, J. M., et al. 2017, A\&A, 602, A33 Freudling, W., Romaniello, M., Bramich, D. M., et al. 2013, A\&A, 559, A96 Gaia Collaboration (Brown, A. G. A., et al.) 2018, A\&A, 616, A1 Garufi, A., Podio, L., Bacciotti, F., et al. 2019, A\&A, 628, A68 Gullbring, E., Hartmann, L., Briceño, C., \& Calvet, N. 1998, ApJ, 492, 323 Hartmann, L. 1998, Accretion Processes in Star Formation (Cambridge University Press)

Herczeg, G., \& Hillenbrand, L. A. 2014, ApJ, 786, 97 Jensen, E. L. N., \& Akenson, R. 2014, Nature, 511, 567 Johns-Krull, C. M. 2007, ApJ, 664, 975

Joncour, I., Duchêne, G., \& Moraux, E. 2017, A\&A, 599, A14 Kraus, A. L., \& Hillenbrand, L. A. 2009, ApJ, 703, 1511

Kurtovic, N. T., Pérez, L. M., Benisty, M., et al. 2018, ApJ, 869, 44 Luhman, K. L., Stauffer, J. R., Muench, A. A., et al. 2003, ApJ, 593, 1093 MacGregor, M. A., Wilner, D. J., Czekala, I., et al. 2017, ApJ, 835, 17 Manara, C. F., Testi, L., Rigliaco, E., et al. 2013, A\&A, 551, A107 Manara, C. F., Frasca, A., Alcalá, J. M., et al. 2017, A\&A, 605, A86 Manara, C. F., Tazzari, M., Long, F., et al. 2019, A\&A, 628, A95 Matthews, T. G., Ade, P. A. R., Angilé, F. E., et al. 2014, ApJ, 784, 116 Mesa, D., Bonnefoy, M., Gratton, R., et al. 2019, A\&A, 624, A4

Monin, J. L., Clarke, C. J., Prato, L., \& McCabe, C. 2007, in Protostars and Planets V, eds. B. Reipurth, D. Jewitt, \& K. Keil (Tucson: University of Arizona Press), 395

Natta, A., Testi, L., Alcalá, J. M., et al. 2014, A\&A, 569, A5

Neuhäuser, R., Guenther, E. W., Wuchterl, G., et al. 2005, A\&A, 435, 13

Nisini, B., Antoniucci, S., Alcalá, J. M., et al. 2018, A\&A, 609, A89

Pecaut, M. J., \& Mamajek, E. E. 2013, ApJS, 208, 9

Petrus, S., Bonnefoy, M., Chauvin, G., et al. 2020, A\&A, 633, A124

Riddick, F., Roche, P., \& Lucas, P. 2007, MNRAS, 381, 1067

Rojas-Ayala, B., Covey, K. R., Muirhead, P. S., \& Lloyd, J. P. 2012, ApJ, 748, 93

Schwarz, H., Ginski, Ch., de Kok, R. J., et al. 2016, A\&A, 593, A74

Shuo, K., Arce, H. G., Maureira, M. J., et al. 2019, ApJ, 874, 104

Stamatellos, D., \& Herczeg, G. J. 2015, MNRAS, 449, 3432

Vernet, J., Dekker, H., D’Odorico, S., et al. 2011, A\&A, 536, A105

Whelan, E. T., Alcalá, J. M., Bacciotti, F., et al. 2014, A\&A, 570, A59

Williams, J. P., Mann, R. K., Di Francesco, J., et al. 2014, ApJ, 796, 120

Wu, Y.-L., Sheehan, P. D., Males, J. R., et al. 2017, ApJ, 836, 223

Zhou, Y., Herczeg, G. J., Kraus, A. L., Metchev, S., \& Cruz, K. L. 2014, ApJ, 783, 17 


\section{Appendix A: Observational strategy and data processing}

The X-shooter science exposure of GQLup consisted in a twocycle (A-B-B-A) nodding mode observation for a total on-source exposure of $1.2 \mathrm{~h}$ using the 1 .'0 slits; as part of the same observing block, an additional short exposure (of $\sim 10 \%$ the science exposure) was performed in stare mode using the wide slits of $5 \prime$.0 immediately before the science observation for slit-loss correction purposes. Such a strategy allows us to achieve a precision in absolute flux calibration on the order of $15-20 \%$. The slit was aligned at parallactic angle.

Data reduction was performed using the X-shooter pipeline version 3.3.5 in the v2.9.1 reflex environment (Freudling et al. 2013). The steps of the data processing are the same as those described in Alcalá et al. (2014, 2017). In particular, the absolute flux calibration was done using the wide-slit short exposure spectrum to correct for slit losses.

\section{Appendix B: Stellar physical parameters}

Spectral type, SpT, was calculated using the spectral indices by Riddick et al. (2007) and the H2O-K2 index from Rojas-Ayala et al. (2012) for the NIR. Extinction is estimated by comparison of the VIS spectrum with that of spectral templates best matching the spectrum of 2MASS J15491331. All the templates have a low extinction $\left(A_{V}<0.5\right)$ and are taken from (Manara et al. 2017, and references therein). The templates were then artificially reddened by $A_{V}$ in the range $0-4.0 \mathrm{mag}$ in steps of $0.25 \mathrm{mag}$, until the best match to the object spectrum was found. The effective temperature, $T_{\text {eff }}$, was estimated using the derived spectral type and the relationship between spectral type and $T_{\text {eff }}$ by Herczeg \& Hillenbrand (2014). Other calibrations (e.g. Luhman et al. 2003; Pecaut \& Mamajek 2013) provide very similar $T_{\text {eff }}$ values.

The Gaia DR2 parallax $(5.49 \pm 0.46$ mas $)$ for $2 \mathrm{MASS}$ J15491331 yields a distance $d=182( \pm 15)$ pc, while for most of the YSOs in Lupus the distance is $159 \mathrm{pc}$ (see appendix in Alcalá et al. 2019). For GQ Lup A, the parallax (6.59 \pm 0.05 mas) yields a distance $d=152( \pm 1)$ pc. The error on the measurement for 2MASS J15491331 is rather large, with the estimatedexcess-noise of 3 mas and astrometric-excess-noise-sig of 59, making it rather unreliable. This is probably due to the edgeon geometry of the object which may affect the photocentre measurement. We note that other objects, such as Sz 102 in Lupus, RY Tau in Taurus (see Garufi et al. 2019), and R CrA (Mesa et al. 2019), with similar edge-on geometries to that of 2MASS J15491331 or extended circumstellar structures suffer from the same DR2 problem. As concluded in Sect. 4, the proximity of 2MASS J15491331 to the GQLup system by a chance projection is very unlikely, with the stars most likely being gravitationally bound. Thus, it is reasonable to adopt the distance of GQ Lup A for 2MASS J15491331, which in any case is different by just $\sim 2 \sigma$.

The bolometric luminosity, $L_{\star}$, was derived by integration of the X-shooter spectrum after correction for extinction using the Cardelli et al. (1989) extinction law and adopting the distance $d=152 \mathrm{pc}$; to include in the integration spectral regions not covered by X-shooter, the spectrum was extrapolated both in the blue and red using the BTSettl model of the same $T_{\text {eff }}$ as 2MASS J15491331. The contribution of this extrapolation to the bolometric flux represents less than $10 \%$ of the total bolometric flux. The stellar radius, $R_{\star}$ was derived from $T_{\text {eff }}$ and $L_{\star}$ using the Stefan-Boltzmann law. We note that $R_{\star}$ is

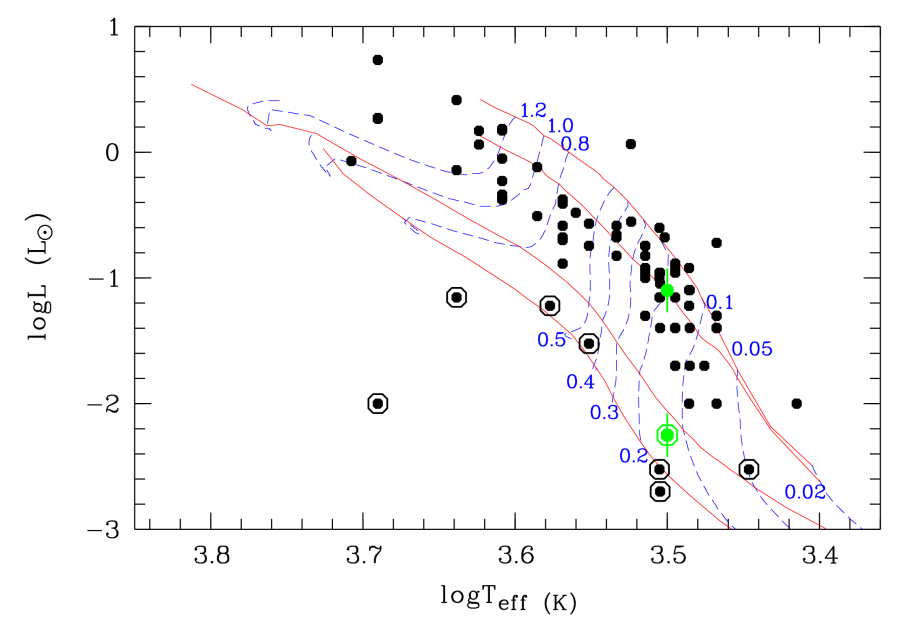

Fig. B.1. HR diagramme of the Lupus sample (black dots) with 2MASS J15491331 overplotted in green. The PMS evolutionary tracks of Baraffe et al. (2015) are overplotted with the dashed blue lines, while isochrones (1 Myr, $3 \mathrm{Myr}, 30 \mathrm{Myr}$ and $10 \mathrm{Gyr}$ ) are the red lines. Subluminous objects are overplotted with encircled dots (2MASS J15491331 in green). The green non-encircled dot is 2MASS J15491331 when luminosity is corrected by disc obscuration.

underestimated for subluminous objects and, as discussed in the main text, 2MASS J15491331 is subluminous with respect to other Lupus members. This can be seen in Fig. B.1 where the position of the star on the HR diagram is shown before and after applying the correction using the luminosity-independent $R_{\star}$ value based on the $\log g$ measurement. The latter has been derived by applying the code ROTFIT (Frasca et al. 2015) to the $\mathrm{X}$-shooter spectrum. The code finds the best photospheric template spectrum that reproduces the target spectrum by minimising the $\chi^{2}$ of the difference between the observed and template spectra in specific spectral segments. The spectral segments are normalised to unity, and therefore a measure of $A_{V}$ is not provided by the code. The spectral intervals analysed with ROTFIT contain features that are sensitive to the effective temperature and/or $\log g$, such as the Na I doublet at $\lambda \approx 819 \mathrm{~nm}$ and the $\mathrm{K}$ I doublet at $\lambda \approx 766-770 \mathrm{~nm}$. The code also allows us to measure the $v \sin i$ by $\chi^{2}$ minimisation applied to spectral segments devoid of broad lines. The results produced by ROTFIT are $T_{\text {eff }}=3230 \pm 101 \mathrm{~K}$, a surface gravity $\log g=3.74 \pm 0.23$, radial velocity $R V=-2.0 \pm 2.8 \mathrm{~km} \mathrm{~s}^{-1}$ and projected rotational velocity $v \sin i=13.0 \pm 6.0 \mathrm{~km} \mathrm{~s}^{-1}$.

Finally, the stellar mass was estimated by comparison with the low-mass PMS evolutionary tracks by Baraffe et al. (2015; see Fig. B.1).

\section{Appendix C: Measurements of mass accretion and ejection rate}

The spectrum of 2MASS J15491331 shows strong emission lines, both permitted and forbidden. The $\mathrm{H} \alpha$ line is intense $(\mathrm{EW} \approx 100 \AA)$ and broad $\left(\Delta \mathrm{V}>500 \mathrm{~km} \mathrm{~s}^{-1}\right)$, the satellite [N II] forbidden lines are seen in emission (see Fig. C.1), and UVexcess emission is evident in the spectrum (Fig. C.2), suggesting high mass accretion and ejection rates.

To derive the accretion luminosity, $L_{\text {acc }}$, we followed the methods described in Alcalá et al. (2014, 2017) and Manara et al. (2017). Briefly, the spectrum of 2MASS J15491331 was fitted as the sum of the photospheric spectrum of a non-accreting template and the emission of a slab of hydrogen; the accretion 


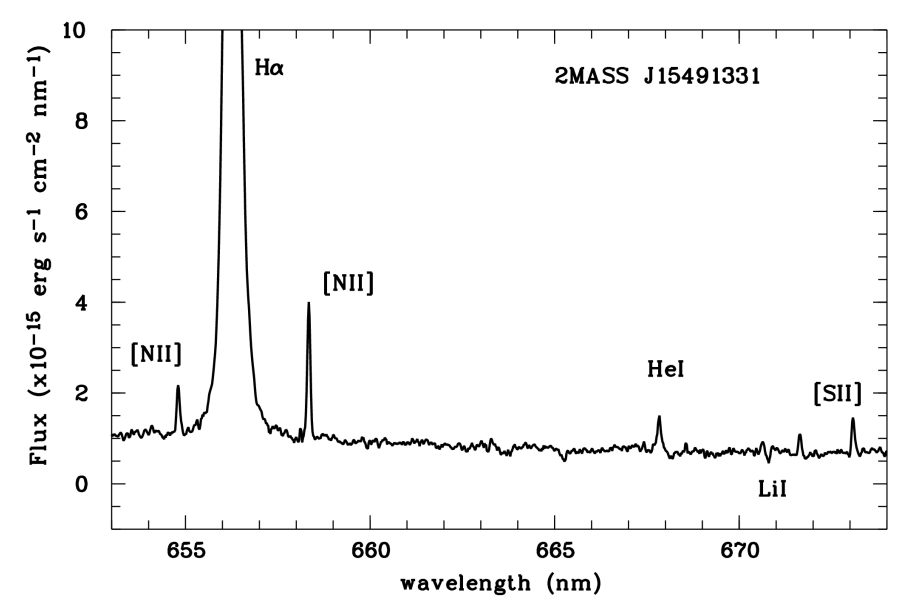

Fig. C.1. Portion of the X-shooter spectrum of 2MASS J15491331 around the $\mathrm{H} \alpha$ line. The $\mathrm{He} \mathrm{I} \lambda 6678 \AA$ and the forbidden lines of [N II] and [S II] and the Li I $\lambda 6708 \AA$ are labelled.

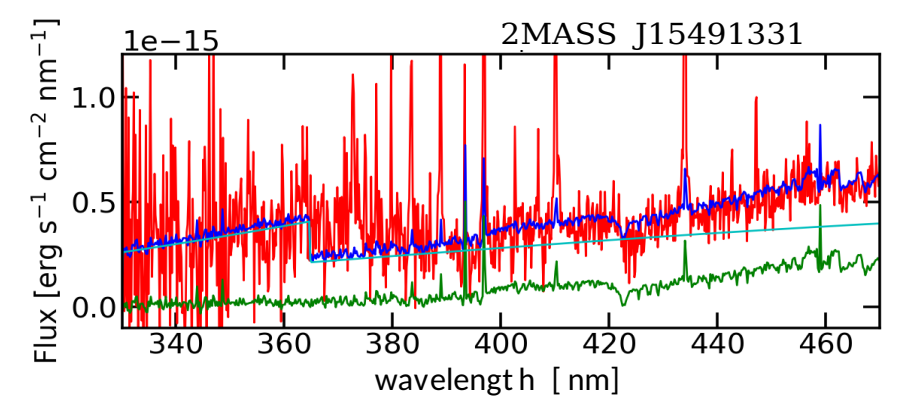

Fig. C.2. Ultraviolet spectrum of 2MASS J15491331 in the region of the Balmer jump (red line). The spectrum of the adopted non-accreting template SO 797 (Manara et al. 2013) is overplotted with a green line. The continuum emission from the slab is shown by the cyan continuous line. The best fit with the emission predicted from the slab model added to the template is given by the blue line.

luminosity is given by the luminosity emitted by the slab. The stellar and accretion parameters are self-consistently derived by finding the best fit among a grid of slab models and using the continuum UV-excess emission and the broad wavelength range covered by the X-shooter spectra (330-2500 nm) to constrain both the spectral type of the target and the interstellar extinction toward it. Figure C. 2 shows the fit. From our analysis we estimate that in general the uncertainty on $L_{\text {acc }}$ is $\sim 0.25$ dex (see Alcalá et al. 2014, 2017; Manara et al. 2017). The best fit yields $L_{\text {acc }}=2.55 \times 10^{-4} L_{\odot}$ or $\log L_{\mathrm{acc}}=-3.6$ and simultaneously the stellar parameters $\mathrm{SpT}=\mathrm{M} 4.5, A_{V}=0.5 \mathrm{mag}, T_{\text {eff }}=3200 \mathrm{~K}$, and $L_{\star}=0.0043 L_{\odot}$. All the results from the fit are highly consistent with those derived in Appendix B, within the errors.

We also used the luminosity, $L_{\text {line }}$, of several permitted emission lines and $L_{\text {line }}-L_{\text {acc }}$ relationships (Alcalá et al. 2017) to derive $L_{\text {acc }}$. The $L_{\text {line }}$ of Balmer lines up to H15, and that of several $\mathrm{He} \mathrm{I}$ and $\mathrm{Ca}$ II permitted lines, were computed by measuring the line fluxes on the $\mathrm{X}$-shooter spectrum, correcting them by extinction $\left(A_{V}=1 \mathrm{mag}\right)$ and adopting the Gaia DR2 distance of GQLup A (see discussion in Appendix B). Figure C.3 shows $L_{\text {acc }}$ as a function of the diagnostics used. Here, $L_{\text {acc }}$ is consistent within errors among the different lines, except for the $[\mathrm{O} I]$ forbidden line. Excluding the latter, the average $\left\langle L_{\mathrm{acc}}\right\rangle$ yields $\log \left\langle L_{\text {acc }}\right\rangle=-3.76$ with a dispersion of 0.20 dex.

The [O I] line, which is also correlated with $L_{\text {acc }}$ (Natta et al. 2014; Nisini et al. 2018), was deliberately included in our

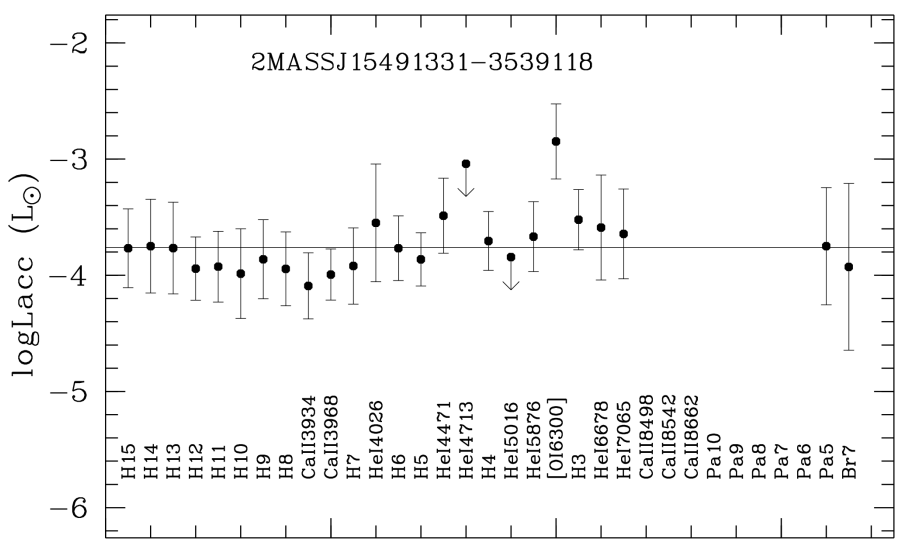

\section{Line}

Fig. C.3. Accretion luminosity as a function of different line diagnostics. The different diagnostics are labelled and sorted by increasing wavelength. The horizontal line corresponds to the average value of $L_{\text {acc. }}$.

analysis because it originates quite far from the star and is free of obscuration from the inner disc. This is why $L_{\mathrm{acc}}$ from this line is about one order of magnitude higher than the values estimated from the permitted lines. The other permitted lines form in the accretion columns in the inner disc regions and hence suffer obscuration by the almost edge-on disc, by a similar amount as the stellar luminosity. Thus, in principle we could estimate a correction of $L_{\text {acc }}$ based on $L_{\text {acc }}$ derived from the [O I] line. A correction of 0.9 dex in $\log L_{\text {acc }}$ can be calculated, and the corrected $\log L_{\text {acc }}$ is -2.84 . This accretion luminosity was converted into mass accretion rate, $\dot{M}_{\text {acc }}$, using the relation

$$
\dot{M}_{\mathrm{acc}}=\left(1-\frac{R_{\star}}{R_{\mathrm{in}}}\right)^{-1} \frac{L_{\mathrm{acc}} R_{\star}}{G M_{\star}} \approx 1.25 \frac{L_{\mathrm{acc}} R_{\star}}{G M_{\star}},
$$

where $R_{\star}$ and $M_{\star}$ are the radius and mass of 2MASS J15491331, respectively, and $R_{\text {in }}$ is the inner-disc radius (Gullbring et al. 1998; Hartmann 1998). The latter corresponds to the distance from the star at which the disc is truncated - due to the stellar magnetosphere - and from which the disc gas is accreted, channelled by the magnetic field lines. It has been found that $R_{\text {in }}$ ranges from $3 R_{\star}$ to $10 R_{\star}$ (Johns-Krull 2007). For consistency with our previous studies (e.g. Alcalá et al. 2014, 2017; Manara et al. 2017, and references therein), here we assumed $R_{\text {in }}$ to be $5 R_{\star}$. We used the ROTFIT corrected $R_{\star}$ (see main text).

Besides the [O I] optical lines, several other forbidden lines are identified in the VIS and UVB arms of the spectrum ([O II], [S II], [N II], see Fig. C.1). None of these lines show evidence of the high-velocity component (HVC) indicative of fast moving jets, but only show the low-velocity component (LVC) that is known to trace slow-moving winds (either thermally or magnetically driven). Following Natta et al. (2014), a rough estimate of the mass loss through the winds traced by the LVC can be obtained assuming a simple spherical geometry for the emitting gas, just dividing the mass of the emitting region by a timescale $\tau=L / v$ where $L$ and $v$ are measured along the flow direction.

In particular, we use the [OI] $\lambda 6300 \AA$ line; that from the flux-calibrated spectrum has a luminosity $L_{[\mathrm{OI}]}=3.65 \times 10^{-7} L_{\odot}$ (assuming a distance of $152 \mathrm{pc}$ ) or a factor 1.4 higher if the distance is 182 pc. From Eq. (6) in Natta et al. (2014) we find that $M_{\text {gas }}=5.11 \times 10^{-12} M_{\odot}$. Moreover, we derive the volume occupied by the gas, and assuming spherical geometry, the linear 
dimension of the region emitting the observed [O I]. We find $L \sim 0.15 \mathrm{AU}$, and assuming a peak velocity of $\sim 1 \mathrm{~km} \mathrm{~s}^{-1}$, this translates into a typical timescale of $\tau \sim 0.8 \mathrm{yr}$. The mass loss rate we obtain is therefore $M_{\text {loss }} \sim 7.0 \times 10^{-12} M_{\odot} \mathrm{yr}^{-1}$ (at $152 \mathrm{pc}$, or $M_{\text {loss }} \sim 8.9 \times 10^{-12} M_{\odot} \mathrm{yr}^{-1}$ at $182 \mathrm{pc}$ ).

\section{Appendix D: Balmer decrements}

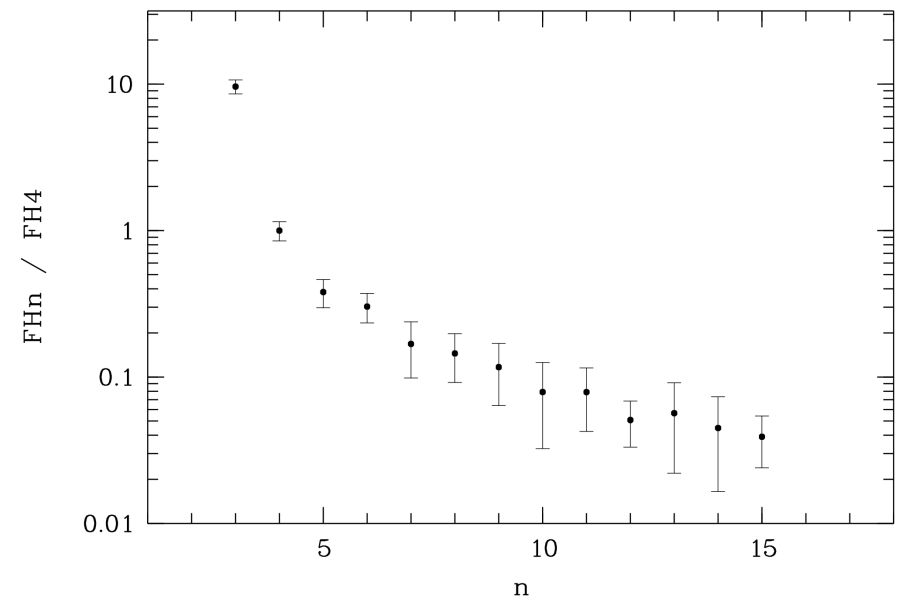

Fig. D.1. Balmer decrements as a function of the quantum number. The $\mathrm{H} \beta$ line has been taken as reference for the analysis.

We also investigated the Balmer decrements in 2MASS J15491331. The results are shown in Fig. D.1. We decided to use $\mathrm{H} \beta$ (or $\mathrm{H} 4$ ) as reference for the same reasons as discussed in Antoniucci et al. (2017). The high $\mathrm{H} \alpha / \mathrm{H} \beta$ ratio implies that the emission is thin in general, and is in agreement with our previous findings for Lupus sources (see Figs. 16 and 17 in Frasca et al. 2017). In that paper we showed that most subluminous sources display large values of the Balmer decrement. Also, the trend of the Balmer decrements in 2MASS J15491331 shows the Type 1 form as defined in Antoniucci et al. (2017). For 2MASS J15491331 $\log (\mathrm{H} 9 / \mathrm{H} 4)=-0.93$, which is consistent with the definition $\log (\mathrm{H} 9 / \mathrm{H} 4)<-0.8$ for Type 1 in Antoniucci et al. (2017). The prototype of this class is the edge-on YSO ParLup3-4, consistent with the edge-on geometry of the 2MASS J15491331 disc.

\section{Appendix E: Rejecting the hypothesis of a chance projection}

The confirmed YSOs (Alcalá et al. 2017; Frasca et al. 2017, and references therein) in Lupus I allowed us to define a range of Gaia DR2 values for $\pi, \mu_{\alpha} \cos \delta$, and $\mu_{\delta}$. However, an important constraint to consider here is that the spatial distribution of the Lupus I members is not uniform, but follows the largescale distribution of the cloud filament. This is more evident for the youngest YSOs (the Class-0 and I proto-stars) detected with the Herschel satellite (Benedettini et al. 2018), which are more concentrated along the filament than the Class-II and III YSOs. We then searched for objects lying in the same parameter space through Gaia DR2 in the Lupus I region, and over an area of $\sim 1.0^{\circ} \times 6.4^{\circ}$ over the Lupus I cloud filament. However, our final Gaia DR2 parameter space is slightly wider than what we found for the actual members. This choice was made for two reasons: to define the sample of candidates sharing similar properties as those of the Class-II and Class-III bona-fide members,

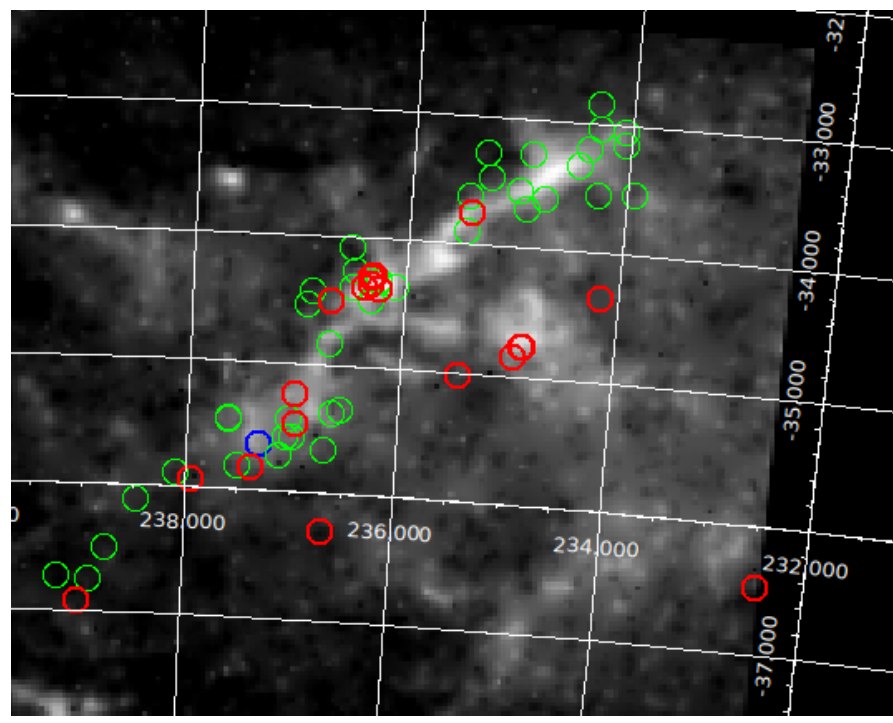

Fig. E.1. Spatial distribution of the selected candidate members from Gaia DR2 (green circles) and the previously known YSOs (red circles) overplotted on the extinction map of the Lupus I filament. The dark blue circle shows the position of the GQLup system. North is up and east to the left.

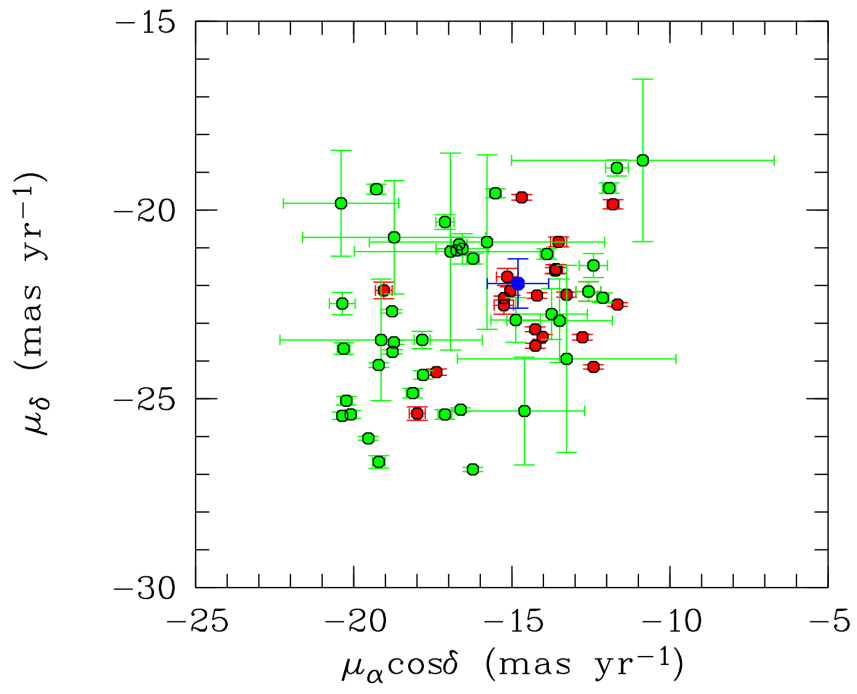

Fig. E.2. Scatter plot of the proper motions of the candidates (green dots) and the previously known YSOs in Lupus I. The position of 2MASS J15491331 is indicated by the dark blue dot. Errors are overplotted.

and to include 2MASS J15491331 in the parameter space despite it lying outside of parallax range (see discussion in Appendix B).

The result is the following: for $5<\pi$ (mas) $<8,-21<$ $\mu_{\alpha} \cos \delta\left(\operatorname{mas~yr}^{-1}\right)<-10,-27<\mu_{\delta}\left(\operatorname{mas~yr}^{-1}\right)<-18$, we found 55 objects in the selected region (see Fig. E.1). These include 42 candidates belonging to Gaia DR2 and 13 previously known members lying on the Lupus I filament. This results in a linear density of 0.0024 objects/arcsec or an average object separation of 418 arcsec, which is 26 times the observed separation between GQ Lup A and 2MASS J15491331. The low chances of finding an object accidentally in the vicinity of GQLup supports the high probability of a physical association to the Lupus I cloud, enhancing the chances of GQ Lup A-B and 2MASS J15491331 being physically bound. This is a robust result as the estimated linear density is only an upper limit, as several of the selected 
candidates are expected to be Sco-Cen members or objects unrelated to Lupus I. In fact, some of the selected candidates appear rather scattered on the $\mu_{\alpha} \cos \delta$ versus $\mu_{\delta}$ scatter plot in comparison with the previously known YSOs (see Fig. E.2).

It is also worth noting that the typical linear density of the proto-stars along the Lupus I cloud filament ( 100/degree, Benedettini et al. 2018) is consistent with an average separation of a few tens of arcsec, implying a few thousand astronomical units at the distance of the Lupus clouds, and on the order of magnitude of the projected separation between GQLup A and 2MASS J15491331. Turbulent fragmentation is expected to occur on a similar scale to the thickness of the filament, which is also on the order of a few thousand astronomical units. All this would support the scenario of turbulent fragmentation along a filament for the formation of the wide binary. We therefore conclude that if 2MASS J15491331 formed within the same filament as GQLup A, then they have a common origin, even though they might be gravitationally unbound. 